\title{
Indicadores de calidad de las harinas de trigo: índice de calidad industrial y su relación con ensayos predictivos
}

\author{
de la Horra A.E.; M.L. Seghezzo, E. Molfese, P.D. Ribotta y A.E. León
}

\begin{abstract}
RESUMEN
El objetivo de este trabajo fue estudiar y evaluar la capacidad de diferentes parámetros para predecir la calidad de las harinas de trigo, analizando las relaciones existentes entre éstos y el índice de calidad industrial (ICI). Se utilizaron siete muestras de harina de trigo provistas por la CEI Barrow. Se determinaron parámetros relacionados con la calidad del grano de trigo, la molienda y la composición de las harinas. Además, se llevaron a cabo ensayos relacionados con el comportamiento de las masas (ensayo farinográfico y alveográfico) y se elaboró pan. Se calculó el $\mathrm{ICl}$ para cada una de las muestras y se estudió su relación con pruebas de predicción y ensayos de calidad. El ICI mostró correlaciones significativas y positivas con el contenido de macropolímero de glutenina, la extensibilidad alveográfica y el tiempo de desarrollo de la masa. Estas relaciones podrían resultar beneficiosas en la evaluación de la aptitud de las harinas para la elaboración de diferentes productos panificados, ya que constituyen determinaciones más sencillas y no requieren de equipamiento de alta tecnología ni grandes cantidades de muestra.
\end{abstract}

Palabras clave: calidad de pan, ensayos predictivos, índice de calidad industrial

de la Horra A.E.; M.L. Seghezzo, E. Molfese, P.D. Ribotta and A.E. León, 2012. Wheat flours quality indicators: industrial quality index and its relation with predictive tests. Agriscientia XXIX (2): 81-89

\section{SUMMARY}

The aim of the present study was to analyze and evaluate the ability of different parameters to predict wheat flours quality, considering the existing relationships between them and the industrial quality index (IQI). Seven wheat flour samples provided by CEI Barrow were used. Parameters related to wheat grain, milling quality and flour composition were established. Dough behavior (farinograph and alveograph tests) and bread quality were determined. The IQI for each sample was calculated and its relationship with predictive and quality tests was evaluated. The IQI showed significant and positive associations with glutenin macropolymer content, alveographic extensibility and dough development 
time. The relationships found may represent an advance for the evaluation of flour suitability to elaborate diverse baking products; as they are simple tests and no high tech equipment or large amounts of sample are required.

Key Words: bread quality, quality predictors, industrial quality index

A.E. de la Horra (ICYTAC, CONICET), P.D. Ribotta (ICYTAC, CONICET) y A.E. León (ICYTAC, CONICET): Facultad de Ciencias Agropecuarias, Universidad Nacional de Córdoba, Avenida Valparaiso y Rogelio Martínez, C.C. 509, 5000, Córdoba, Argentina. M.L. Seghezzo y E. Molfese: Laboratorio de Calidad Industrial de Granos, Chacra Experimental Integrada Barrow (Convenio INTA-MAA), CP 7500, Buenos Aires, Argentina. Correspondencia a A.E. León: aeleon@agro.unc.edu.ar

\section{INTRODUCCIÓN}

El trigo es uno de los tres cereales más cultivados en el mundo, junto al maíz y el arroz. Se lo utiliza para la producción de harinas y sémolas a partir de las cuales se obtienen una gran variedad de productos alimenticios como pan, galletitas, pastas y otros. En la Argentina se consumen un promedio de 9,6 kg de bizcochos y galletitas (Lezcano, 2010) y $72,5 \mathrm{~kg}$ de pan por habitante por año, lo que la posiciona dentro de América como uno de los principales países consumidores de productos panificados elaborados con harina de trigo (Lezcano, 2007).

Las panificadoras industriales necesitan distintos tipos de harina para confeccionar, en procesos altamente automatizados, los diferentes productos panificados (Otamendi, 2004). Al mismo tiempo, la innovación en este sector se vincula con harinas diferenciadas de acuerdo a su destino industrial, denominadas especiales y pre-mezclas, que permiten obtener determinadas características reológicas y propiedades viscoelásticas de las masas (Cuniberti y Menella, 2004). La clasificación de los trigos resulta fundamental para satisfacer la demanda de la industria y de la exportación (Cuniberti, 2011), de manera tal de proveer un producto de idénticas características en forma consistente y homogénea en el tiempo (Otamendi, 2004) y de una calidad específica según el destino final (Cuniberti y Menella, 2004). El cumplimiento de este objetivo permitiría aumentar la credibilidad y la confiabilidad de Argentina en el comercio mundial como país exportador de trigo (Cuniberti, 2011).

La definición de la calidad de los trigos y las harinas es un elemento clave para garantizar la obtención del producto esperado. Sin embargo, pese a la cantidad de trabajos destinados a su determinación, es un concepto que permanece en debate. La caracterización físico-química y reológica de las harinas en estudio y la elaboración de un producto panificado a partir de éstas es la forma ideal de establecer su calidad (Quaglia, 1991). En la Argentina se ha propuesto el índice de calidad industrial (ICI) (Salomón y Miranda, 2003), que presenta la ventaja de combinar indicadores de calidad molinera y de calidad panadera; pero no incluye otros parámetros de composición de las harinas, que son importantes debido a los efectos que poseen sobre sus propiedades funcionales.

En muchas ocasiones no es posible elaborar un producto panificado, ya que es necesario contar con información sobre la calidad de una harina de manera inmediata o no se dispone de la suficiente cantidad de muestra. También es común que no se cuente con los equipos necesarios para obtener los parámetros reológicos que permitan calcular el Índice de Calidad Industrial.

En estos casos, la utilización de ensayos predictivos que se relacionan específicamente con la aptitud de las harinas para elaborar diferentes productos, constituye una opción. Dentro de los ensayos sugeridos se encuentra el índice de sedimentación en dodecil sulfato de sodio (ISSDS), que determina la capacidad de hidratación y de expansión de las proteínas del gluten en un medio ligeramente ácido (Axford et al., 1979). Otra de las pruebas utilizadas es la capacidad de retención de solventes (SRC) (AACC, 2000). Ésta evalúa la capacidad de una harina para retener cuatro solventes diferentes (agua, sacarosa 50\%, carbonato de sodio 5\% y ácido láctico 5\%) (Guttieri et al., 2002). Los valores de cada solvente retenido se relacionan con la presencia de distintos componentes de la harina y permiten generar un 
perfil de calidad y funcionalidad, útil para predecir su aptitud panadera.

El objetivo del presente trabajo fue estudiar la capacidad de diferentes parámetros -reológicos, físico-químicos y del producto panificado- para predecir la calidad de las harinas de trigo. Para tal fin se analizaron las relaciones existentes entre los ensayos predictivos, la composición de las harinas y el índice de calidad industrial.

\section{MATERIALES Y MÉTODOS}

\section{Materiales}

Se emplearon siete muestras de harina de trigo de diferentes cultivares, provistas por la Chacra Experimental Integrada Barrow (Convenio INTAMAA). Los granos fueron molidos en un molino experimental Buhler 202 D (Buhler, Suiza).

\section{Parámetros de calidad del grano de trigo y la molienda}

El peso hectolítrico de los granos de trigo se calculó según la Norma ISO 7971, 1995 y se expresó en $\mathrm{kg} / \mathrm{hl}$. El peso de mil granos se determinó siguiendo la Norma IRAM 15853, 2012. El rendimiento de las harinas se obtuvo según la Norma IRAM 15854, 2010 parte I y II. La relación molinera se calculó como el cociente entre el rendimiento de las harinas y el contenido de cenizas de las muestras.

\section{Composición y caracterización de las harinas}

El contenido de humedad de las muestras se determinó sobre 2,000 $\pm 0,0005 \mathrm{~g}$ de harina, calentando la muestra a $135 \pm 1{ }^{\circ} \mathrm{C}$ durante $2 \mathrm{~h}$ (Método 44-19, AACC, 1995). El contenido de cenizas y proteínas fue determinado según AACC 08-01 y 46-10, respectivamente (AACC, 2000). La cuantificación de pentosanos totales y pentosanos solubles se realizó según la metodología descripta en Roccia et al. (2006). La determinación de gluten húmedo y seco se realizó mediante el método de lavado automático (Norma IRAM 15864, 2007) y manual respectivamente (Método 38-10, AACC, 1995). El macropolímero de glutenina (GMP) se obtuvo según Don et al. (2003) y su contenido de proteínas se determinó por el método de Kjeldahl (Método 46-10, AACC, 2000).

Se determinó el número de caída de las harinas según la Norma IRAM 15862, 2003. Las determinaciones se realizaron utilizando un equipo
Falling Number 1400 (Perten, Suecia) y los valores registrados se expresaron en segundos.

El ensayo alveográfico se realizó según la Norma IRAM 15857, 1995. Se preparó una masa, de contenido de humedad constante, a partir de la muestra de harina y de una solución de cloruro de sodio 2,5\% p/v, bajo ciertas condiciones de temperatura $\left(24{ }^{\circ} \mathrm{C}\right)$ y amasado en un alveógrafo Chopin tipo MA 95 (Trippette \& Renaud, Francia). Luego de un proceso de insuflado con aire se generaron cinco gráficos, a partir del los cuales se obtuvo una curva promedio. Sobre ésta última se calcularon los parámetros que indican la tenacidad $(P)$, extensibilidad $(L)$, la relación $P / L$ y la fuerza o trabajo (W) necesario para insuflar la masa.

El comportamiento de la masa durante el amasado se evaluó utilizando un farinógrafo Brabender (C.W. Brabender Instruments, Inc., Alemania) según la Norma IRAM 15855, 2000. Se colocó en una amasadora una determinada cantidad de agua y harina y se amasó a velocidad constante, registrando la resistencia que opone la masa al trabajo mecánico continuo en función del tiempo. Primero se determinó la capacidad de absorción de agua para que la masa alcance una consistencia determinada (500 unidades Brabender). Luego, a partir del diagrama resultante, se obtuvieron los siguientes parámetros que se relacionan con la aptitud industrial de la harina: capacidad de absorción de agua (AA), tiempo de desarrollo de la masa (TD), estabilidad de la masa (EST) y aflojamiento de la masa (AFLO).

\section{Panificación}

Los ensayos de panificación se llevaron a cabo siguiendo la Norma IRAM 15858-1, 1996. La formulación de la masa consistió en $100 \%$ de harina, 3\% de levadura prensada, 2,5\% de azúcar, $1 \%$ de sal y agua (el agua a agregar se calculó según el valor $\mathrm{W}$ del ensayo alveográfico). Los ingredientes fueron mezclados en una amasadora, y se llevó la masa formada a fermentar durante 40 minutos, en una cámara a $30{ }^{\circ} \mathrm{C}$ y $70 \%$ de humedad relativa. Luego se sometió a la masa a un proceso de desgasificación manual y posterior fermentación durante 20 minutos. La masa fue laminada en una sobadora pasándola tres veces en distintas posiciones $(3,8 \mathrm{~cm}, 0,6 \mathrm{~cm}$ y 0,4 $\mathrm{cm}$ ), se le dio forma de rollo con una armadora y se la colocó en un molde. El molde fue llevado a la cámara de fermentación por 75 minutos. La cocción se realizó a $225{ }^{\circ} \mathrm{C}$ durante 30 minutos. Se registró el volumen (VOL) de cada pieza y se las pesó para calcular el volumen específico (Ve). 


\section{Índice de calidad industrial}

El índice de calidad industrial se calculó como la suma ponderada de las siguientes variables: peso hectolítrico, porcentaje de proteína en grano, porcentaje de gluten húmedo, relación molinera, fuerza de la masa obtenida del alveograma, estabilidad farinográfica y volumen de pan (Salomón y Miranda, 2003).

$$
\begin{aligned}
& I C I=(0,075 * P H+0,075 * P r o t+0,15 * R E L M O L+ \\
& 0,1 * G \boldsymbol{h}+0,25 * W+0,10 * E S T+0,30 * V O L) * 10
\end{aligned}
$$

$\mathrm{PH}$ : peso hectolítrico, Prot: contenido de proteínas, REL MOL: relación molinera, Gh: contenido de gluten húmedo, W: fuerza alveográfica, EST: estabilidad farinográfica, VOL: volumen de pan.

\section{Pruebas de predicción y ensayos de calidad de harinas.}

Índice de sedimentación en dodecil sulfato de sodio (IS-SDS) (Método 56-70, AACC, 1995): se determinó midiendo el volumen en $\mathrm{cm}^{3}$ que se obtuvo a partir de $1,0 \mathrm{~g}$ de harina, la cual fue humectada con una solución de SDS al 2\% - ácido láctico 1:8 v/v y sometida a sucesivos períodos de agitación y reposo.

Perfil de capacidad de retención de solventes (SRC) (Método 56-11, AACC, 2000): a cuatro porciones individuales de $5,0 \mathrm{~g}$ de muestra se las hidrató por separado con $25 \mathrm{~g}$ de las siguientes soluciones: agua destilada, ácido láctico 5\% (p/p), sacarosa $50 \%(p / p)$ y carbonato de sodio $5 \%(p / p)$. Luego de agitación con posterior centrifugación para descartar el sobrenadante, se obtuvo la masa de cada porción hidratada y se calculó el porcentaje de SRC.

\section{Análisis estadístico}

En todos los casos se realizaron las determinaciones por duplicado y los resultados se informaron como el valor promedio. Las relaciones entre los parámetros medidos fueron evaluadas por el método de Pearson (nivel de significación de $0,1)$. Se llevó adelante un análisis de componentes principales con el fin de ponderar el peso de ciertas variables sobre las componentes principales obtenidas y estudiar la variabilidad entre las muestras. Todos los análisis se realizaron mediante el programa estadístico INFOSTAT (Balzarini et al., 2008; Di Rienzo et al., 2008).

\section{RESULTADOS Y DISCUSIÓN}

Los granos analizados presentaron valores de peso hectolítrico entre 70,30 y $81,05 \mathrm{~kg} / \mathrm{hl}$ y el peso de mil granos varió entre 27,10 y 33,60 g (Tabla 1). El rendimiento de las harinas en estudio fue superior al $65,5 \%$. La relación molinera varió entre 120,18 y $152,61 \%$, lo que indica que los granos tuvieron comportamientos diferentes durante el proceso de molienda.

Tabla 1: Parámetros de calidad del grano de trigo y la molienda.

\begin{tabular}{ccccc}
\hline Muestras & $\begin{array}{c}\mathrm{PH} \\
(\mathrm{Kg} / \mathrm{hl})\end{array}$ & $\begin{array}{c}\text { PMG } \\
(\mathrm{g})\end{array}$ & $\begin{array}{c}\text { Rendimiento } \\
(\%)\end{array}$ & $\begin{array}{c}\text { Rel. Mol. } \\
(\%)\end{array}$ \\
\hline 1 & 70,30 & 27,3 & 70,1 & 133,8 \\
2 & 74,55 & 28,7 & 66,8 & 124,6 \\
3 & 76,35 & 27,9 & 73,1 & 152,6 \\
4 & 73,20 & 27,1 & 66,2 & 123,3 \\
5 & 81,05 & 32,2 & 70,8 & 123,6 \\
6 & 73,20 & 28,9 & 65,5 & 120,2 \\
7 & 77,00 & 33,6 & 70,0 & 150,5
\end{tabular}

PH: peso hectolítrico, PMG: peso de mil granos, Rendimiento: rendimiento de la harinas, Rel. Mol.: relación molinera.

El contenido de cenizas de las harinas fue inferior al $0,64 \%$ (Tabla 2). El porcentaje de proteínas

Tabla 2: Contenido de cenizas, proteína, gluten húmedo (Gh), gluten seco (Gs), pentosanos totales (PT), pentosanos solubles (PS), almidón dañado (AD), macropolímero de gluteninas (GMP) y numero de caída (FN).

\begin{tabular}{cccccccccc}
\hline Muestras & $\begin{array}{c}\text { Cenizas } \\
(\%)\end{array}$ & $\begin{array}{c}\text { Proteína } \\
(\%)\end{array}$ & $\begin{array}{c}\text { Gh } \\
(\%)\end{array}$ & $\begin{array}{c}\text { Gs } \\
(\%)\end{array}$ & $\begin{array}{c}\text { PT } \\
(\%)\end{array}$ & $\begin{array}{c}\text { PS } \\
(\%)\end{array}$ & $\begin{array}{c}\text { AD } \\
(\%)\end{array}$ & $\begin{array}{c}\text { GMP } \\
(\%)\end{array}$ & $\begin{array}{c}\text { FN } \\
(\mathrm{seg})\end{array}$ \\
\hline 1 & 0,60 & 16,05 & 40,1 & 13,1 & 7,97 & 1,74 & 6,4 & 4,83 & 343 \\
2 & 0,64 & 16,29 & 36,2 & 12,1 & 7,92 & 2,04 & 8,4 & 4,99 & 439 \\
3 & 0,54 & 12,23 & 28,2 & 9,4 & 7,51 & 1,53 & 7,6 & 4,66 & 420 \\
4 & 0,59 & 13,76 & 34,1 & 10,8 & 7,88 & 2,09 & 6,9 & 4,18 & 433 \\
5 & 0,61 & 11,60 & 30,8 & 9,8 & 7,71 & 2,09 & 10,5 & 4,19 & 413 \\
6 & 0,41 & 12,00 & 30,8 & 10,3 & 6,53 & 2,21 & 9,3 & 3,81 & 369 \\
7 & 0,49 & 16,09 & 42,2 & 13,9 & 7,46 & 1,76 & 8,9 & 5,72 & 432 \\
\hline
\end{tabular}

Los valores son expresados en base seca (g/g sólidos). 
totales osciló entre 11,60 y 16,29\%. Los porcentajes de gluten húmedo y seco fueron similares a los informados por Kulkarni et al. (1987) para harinas de trigos Hard Red Winter. Se encontró una fuerte asociación entre estos últimos porcentajes y el contenido de proteínas $(r=0,90$ y $r=0,92 ; p<0,1)$, lo que indica que cuanto mayor es el porcentaje de proteínas totales más alto es el contenido de las proteínas en la red de gluten; coincidiendo con Steffolani et al. (2007). El porcentaje de pentosanos totales varió entre 6,53 y $7,97 \%$ y el de pentosanos solubles entre 1,53 y $2,21 \%$, resultados similares a los encontrados por Moiraghi et al. (2011) y Delcour et al. (1989) para harinas de trigos blandos y centeno, respectivamente. El porcentaje de almidón dañado en las harinas osciló entre 6,4 y 10,5\%. La variación en estos valores es muy importante, ya que los gránulos de almidón que sufren daños físicos durante la molienda absorben una mayor cantidad de agua, en algunos casos hasta un $100 \%$ de su peso, en comparación con aquellos que permanecen intactos. Además, los gránulos de almidón dañado son más susceptibles al ataque de las $\alpha$ amilasas que actúan durante el proceso de panificación (Drapron \& Godon, 1987).

El GMP es un gel insoluble en SDS, compuesto de agregados proteicos de gran tamaño, constituido principalmente por unidades de gluteninas (Don et al., 2003). El contenido de GMP de las muestras osciló entre 3,81 y $5,72 \%$. Estos valores resultaron mayores a los informados por Don et al. (2006), lo cual revela una mayor calidad proteica de las harinas que las estudiadas por estos autores. Se observó una correlación positiva entre el contenido de GMP y los porcentajes de proteínas totales, gluten húmedo y seco $(r=0,78 ; r=0,75 ; r=0,78$; $\mathrm{p}<0,1)$.

La Tabla 3 presenta los valores de las determinaciones farinográficas, alveográficas y la calidad de pan. La $L$ de las masas aumentó con el incremento del contenido proteico de las harinas $(r=0,84 ; p<0,1)$; éste a su vez presentó una correlación negativa con la relación $P / L(r=-0,78$; $p<0,1)$. La misma tendencia fue observada por Edwards et al. (1999) y Cuniberti et al. (2003). Por otro lado, se encontraron correlaciones positivas entre la $L$ de las muestras y el contenido de gluten húmedo y seco $(r=0,96$ y $r=0,94 ; p<0,1)$. No se observaron correlaciones significativas entre la $\mathrm{P}$, el $\mathrm{W}$ y los parámetros relacionados con la composición de las harinas. La muestra 5, con un bajo contenido proteico, presentó un valor relativamente alto para W y \%SRC láctico, lo cual puede asociarse a una fracción de gluteninas de buena calidad.

El índice de sedimentación en dodecil sulfato de sodio (IS-SDS) se utiliza como indicador de la calidad panadera de las harinas en estudio. Mientras más alto sea el índice más apta será la harina para la elaboración del pan (Amaya \& Peña, 1991). Los valores obtenidos (Tabla 4) variaron entre 18,00 y $19,25 \mathrm{~cm}^{3}$ y son más altos que los registrados para trigos blandos (Guttieri et al., 2004) y para triticale (Roccia et al., 2006). La muestra 7 , con un gran contenido de proteínas, presentó valores altos para el IS-SDS y el Ve. Mientras que la muestra 5, con bajo porcentaje de proteínas, obtuvo un IS-SDS bajo y un Ve alto. Esto revela la

Tabla 4: Perfil de capacidad de retención de solventes e IS-SDS de las harinas analizadas.

\begin{tabular}{cccccc}
\hline Muestras Sacarosa & $\begin{array}{c}\text { Láctico } \\
(\%)\end{array}$ & $\begin{array}{c}\text { Agua } \\
(\%)\end{array}$ & $\begin{array}{c}\text { Carbonato } \\
(\%)\end{array}$ & $\begin{array}{c}\text { IS-SDS } \\
\left(\mathrm{cm}^{3}\right)\end{array}$ \\
\hline 1 & 104,4 & 114,7 & 60,8 & 78,3 & 18,55 \\
2 & 116,8 & 134,4 & 70,0 & 92,6 & 18,50 \\
3 & 98,6 & 129,8 & 61,0 & 76,5 & 18,75 \\
4 & 107,2 & 130,9 & 63,0 & 84,3 & 18,75 \\
5 & 123,1 & 134,1 & 67,1 & 87,0 & 18,00 \\
6 & 133,7 & 143,3 & 71,7 & 107,8 & 19,25 \\
7 & 129,9 & 169,7 & 66,9 & 89,3 & 18,75 \\
\hline
\end{tabular}

Tabla 3: Parámetros alveográficos, farinográficos y de panificación.

\begin{tabular}{|c|c|c|c|c|c|c|c|c|c|c|}
\hline \multirow[b]{2}{*}{ Muestras } & \multicolumn{4}{|c|}{ Alveograma } & \multicolumn{4}{|c|}{ Farinograma } & \multicolumn{2}{|c|}{ Panificación } \\
\hline & $\begin{array}{c}\mathrm{P} \\
(\mathrm{mm})\end{array}$ & $\begin{array}{c}\mathrm{L} \\
(\mathrm{mm})\end{array}$ & $\begin{array}{c}W \\
\left(\mathrm{~J} \times 10^{4}\right)\end{array}$ & $P / L$ & $\begin{array}{l}\text { AA } \\
(\%)\end{array}$ & $\begin{array}{c}\text { TD } \\
(\min )\end{array}$ & $\begin{array}{c}\text { EST } \\
\text { (min) }\end{array}$ & $\begin{array}{l}\text { AFLO } \\
\text { (min) }\end{array}$ & $\begin{array}{l}\text { Vol } \\
(\mathrm{ml}) \\
\end{array}$ & $\begin{array}{c}\mathrm{Ve} \\
(\mathrm{ml} / \mathrm{g})\end{array}$ \\
\hline 1 & 66 & 139 & 299 & 0,48 & 57,2 & 9,3 & 12,2 & 61 & 580 & 4,1 \\
\hline 2 & 123 & 117 & 536 & 1,05 & 63,6 & 22,3 & 14,7 & 57 & 635 & 4,4 \\
\hline 3 & 84 & 68 & 235 & 1,23 & 55,6 & 2,2 & 29,4 & 3 & 610 & 4,2 \\
\hline 4 & 92 & 114 & 367 & 0,81 & 58,7 & 28,8 & 31,9 & 63 & 670 & 4,7 \\
\hline 5 & 102 & 84 & 320 & 1,22 & 58,1 & 13,9 & 39,0 & 18 & 660 & 4,7 \\
\hline 6 & 112 & 69 & 262 & 1,62 & 62,4 & 5,5 & 12,1 & 42 & 600 & 4,0 \\
\hline 7 & 79 & 185 & 460 & 0,43 & 61,2 & 23,5 & 19,9 & 59 & 800 & 5,7 \\
\hline
\end{tabular}

P: tenacidad, L: extensibilidad, W: trabajo necesario para amasar la masa, AA: capacidad de absorción de agua, TD: tiempo de desarrollo de la masa, EST: estabilidad de la masa, AFLO: aflojamiento de la masa, Vol: volumen de cada pieza, Ve: Volumen específico. 
relación entre el IS-SDS y la calidad y cantidad de las proteínas, en particular las gluteninas.

El perfil SRC de las harinas estudiadas se observa en la Tabla 4. El porcentaje de SRC de cada solvente se relaciona con los componentes de la harina; de esta manera pueden compararse con patrones de comportamiento que permiten clasificar a una determinada harina por su aptitud para desarrollar ciertos productos de panificación, y definir su perfil de calidad. El \%SRC sacarosa y el \%SRC carbonato de sodio se asocian directamente con el contenido de pentosanos y gliadinas y con los niveles de almidón dañado respectivamente. El \%SRC ácido láctico y el \%SRC agua están relacionados con la fracción del gluten correspondiente a las gluteninas y los componentes hidrofílicos (almidón dañado, proteínas totales, gluten y pentosanos solubles) presentes en la harina (Gaines, 2000).

El \%SRC sacarosa correlacionó con el \%SRC carbonato, el \%SRC láctico y el \%SRC agua $(r=0,85 ; r=0,73 ; r=0,86$ con $p<0,1)$. La correlación positiva entre el \%SRC acido láctico y el \%SRC sacarosa también fue encontrada para trigos blandos en otros trabajos (Guttieri et al., 2002; Guttieri \& Souza, 2003), en donde se sugirió que la asociación alcanzada podría ser debida a características propias de dos de las líneas en estudio, más que a una propiedad universal de los trigos o de los solventes SRC. La correlación observada entre \%SRC sacarosa y el \%SRC carbonato de sodio coincide con lo informado en trabajos anteriores para trigos blandos (Guttieri \& Souza, 2003) y triticale (Roccia et al., 2006). No se encontró correlación entre el \%SRC acido láctico y el contenido total de proteínas, lo que concuerda con lo reportado por Guttieri et al. (2001) para trigos blandos. Tampoco se observó asociación entre el \%SRC acido láctico y el \%SRC agua, lo que puede reflejar que la capacidad de retener agua en las harinas analizadas no depende de las características de las proteínas del gluten. La correlación positiva entre el \%SRC láctico y el Ve $(r=0,78 ; p<0,1)$ muestra la influencia de la fracción de gluteninas en la calidad final de la pieza de pan, que está en gran medida dada por el volumen que ésta pueda lograr al final del horneado. Las correlaciones positivas entre \%SRC agua y el contenido de almidón dañado $(r=0,72$; $p<0,1)$ y pentosanos solubles $(r=0,71 ; p<0,1)$ sugieren que son los principales componentes hidrofílicos responsables de la absorción de agua de las harinas. La muestra 1 presentó los menores contenidos de AD y de \%SRC agua; por el contrario, la muestra 5 tuvo los mayores valores de estos parámetros.
Los $\mathrm{ICl}$ calculados para las harinas en estudio (Tabla 5) oscilaron entre 34,8 y 82,0 . Un rango de variación más estrecho fue informado por Abbate et al. (2010) para cultivares de ciclo corto y largo.

Tabla 5: Índice de calidad industrial (ICI)

\begin{tabular}{cc}
\hline Muestras & $\mid \mathrm{Cl}$ \\
\hline 1 & 51,0 \\
2 & 60,9 \\
3 & 42,7 \\
4 & 60,6 \\
5 & 60,5 \\
6 & 34,8 \\
7 & 82,0 \\
\hline
\end{tabular}

Figura 1: Relación del $\mathrm{ICl}$ con el contenido de GMP, la extensibilidad (L) y el tiempo de desarrollo de la masa (TD).
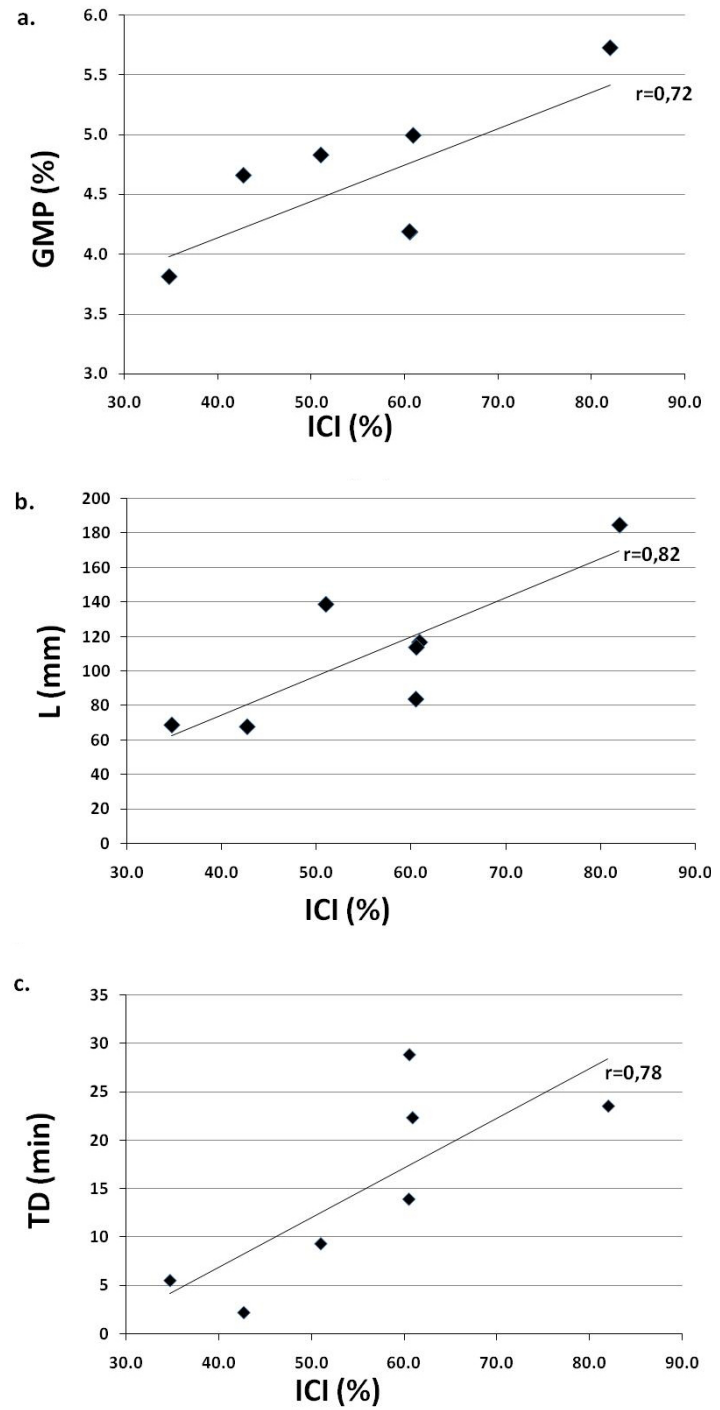
El ICl constituye un buen indicador de la calidad de una harina, reúne las variables más importantes y permite una rápida comparación con variedades de aptitud molinera y harinera conocidas (Salomón y Miranda, 2003). Sin embargo, es importante encontrar determinaciones más sencillas que presenten una significativa asociación con la aptitud de las harinas para la elaboración de determinados productos finales. Se observó una correlación positiva entre el contenido de GMP y el ICl $(r=0,72 ; p<0,1)$ (Figura 1, a). Esto posiciona a la determinación del contenido de GMP como un ensayo preliminar para establecer la calidad de una harina en estudio en casos donde no se cuente con los recursos o el tiempo suficientes para realizar las determinaciones que conforman al $\mathrm{ICl}$. Este resultado está en consonancia con lo informado por Weegels et al. (1996, 1997). El contenido de GMP muestra una alta asociación con el volumen de pan, lo que sugiere que el contenido de GMP de una muestra de harina se vuelve un parámetro de calidad importante para predecir el comportamiento de la harina durante la panificación.

Otro parámetro con el que el $\mathrm{ICl}$ mostró una fuerte correlación fue la $L$ alveográfica $(r=0,82$; $p<0,1$ ) (Figura 1, b). Este resultado corrobora que la $L$ es un valor significativo y útil para caracterizar a una harina, fundamentalmente cuando se trata de trigos argentinos, que se destacan por su tenacidad. A su vez, estos dos parámetros, GMP y $L$, correlacionaron entre sí por lo que se podría decir que el análisis de las propiedades del GMP permite predecir en parte el comportamiento de la masa. Además, se observó una correlación positiva entre el ICl y el TD de la masa $(r=0,78$; $p<0,1$ ) (Figura 1, c). El TD permite describir el comportamiento de la masa y otorga información acerca de las características de la harina, como el tiempo que ésta requiere durante el amasado hasta llegar a formar la red de gluten. Por otro lado, el $\mathrm{ICI}$ no presentó correlaciones significativas con las

Figura 2: Análisis de componentes principales

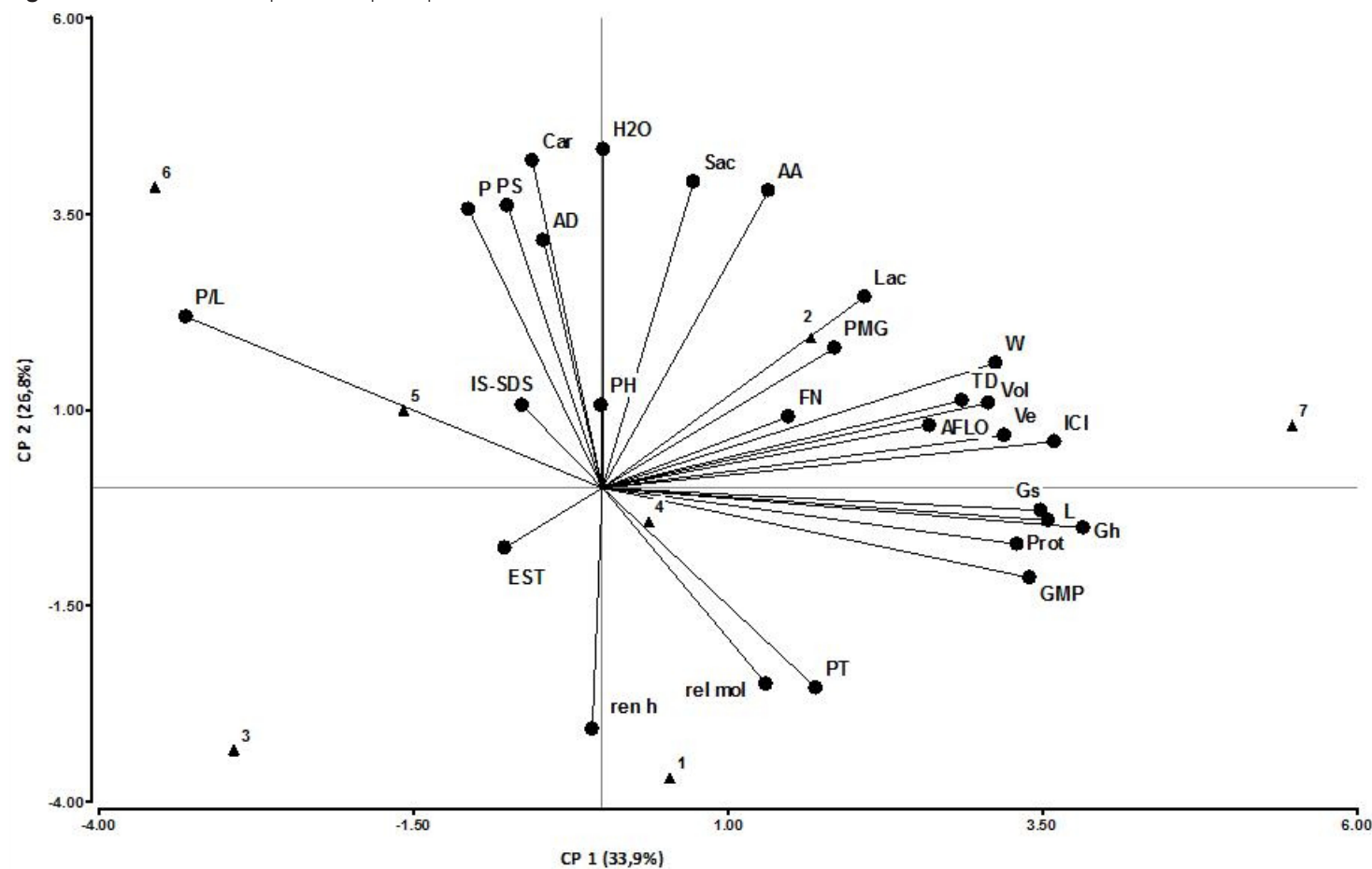

CP: componente principal, $(\bullet)$ : ICl: índice de calidad industrial, Ve: Volumen específico, AFLO: aflojamiento de la masa, TD: tiempo de desarrollo de la masa, Vol: volumen de cada pieza, W: trabajo necesario para amasar la masa, FN: numero de caída, PMG: peso de mil granos, Lac: \% SRC ácido láctico, AA: capacidad de absorción de agua, Sac: \% SRC Sacarosa, H2O: \% SRC agua, Car: \% SRC Carbonato, AD: almidón dañado, PS: pentosanos solubles, PH: peso hectolítrico, P: tenacidad, IS-SDS: índice de sedimentación en SDS, H: humedad, EST: estabilidad de la masa, ren h: rendimiento de la harina, Cen: cenizas, rel mol: relación molinera, PT: pentosanos totales, GMP: macropilímero de glutenina, Prot: contenido de proteínas, Gh: gluten húmedo, L: extensibilidad, Gs: gluten seco. (• ): muestras $1,2,3,4,5,6$ y 7 . 
pruebas de predicción y los ensayos de calidad.

La Figura 2 muestra el análisis de componentes principales para las variables en estudio. El GMP y la $L$ presentaron altos coeficientes de ponderación para la componente principal 1 (CP1) $(0,27$ y 0,31 respectivamente), al igual que otras variables que se utilizan en el cálculo del ICl, como el contenido de proteínas $(0,27)$ y el gluten húmedo $(0,29)$. Estas observaciones resultan importantes, ya que los dos primeros ejes obtenidos (CP1 y CP2) explican el $60,7 \%$ de la variabilidad entre las muestras. Se aprecian correlaciones positivas entre el TD, el GMP, la extensibilidad y el ICI (ángulos menores a $90^{\circ}$ entre los vectores asociados a cada variable).

El contenido de GMP en las harinas y la extensibilidad de las masas constituyen buenos parámetros para la caracterización físico-química y la clasificación de las harinas en estudio. Las significativas correlaciones encontradas entre éstos y el $\mathrm{ICl}$ revelan la relación existente con la aptitud de las harinas para elaborar diferentes productos. Esto puede resultar beneficioso en casos donde la disponibilidad de equipamiento, la cantidad de muestra y/o el tiempo necesarios para realizar los ensayos constituyen un problema.

\section{BIBLIOGRAFÍA}

AACC (American Association of Cereal Chemists), 1995. Approved Methods of the AACC. 9th Edition, AACC, Inc.: St. Paul Minessota.

AACC (American Association of Cereal Chemists), 2000. Approved Methods of the AACC. 10th Edition, AACC, Inc.: St. Paul Minessota.

Abbate, P.E.; F. Gutheim, O. Polidoro, H.J. Milisich y M. Cuniberti, 2010. Fundamento para la clasificación del trigo argentino por calidad: efectos del cultivar, la localidad, el año y sus interacciones. Agriscientia XXVII: $1-9$.

Amaya, A. and R.J. Peña, 1991. Triticale industrial quality improvement at CIMMYT: past, present and future, In: Proceedings of the $2^{\text {nd }}$ International Triticale Symposium, Brasil. pp. 412-421.

Axford, D.W.E.; E.E. McDermott and D.G. Redman, 1979. Note on the Sodium Dodecyl Sulfate Test of Breadmaking Quality: Comparison with Pelshenke and Zeleny Tests. Cereal Chemistry 56:582-584.

Balzarini, M.G.; L. Gonzalez, M. Tablada, F. Casanoves, J.A. Di Rienzo y C.W. Robledo, 2008. INFOSTATManual del Usuario, segunda edición. Córdoba, Argentina. Editorial Brujas, pp.144-145 y 182-185.

Cuniberti, M.B.; M.R. Roth and F. MacRitchie, 2003. Protein Composition-Functionality Relationships for a
Set of Argentinean Wheats. Cereal Chemistry 80:132134.

Cuniberti, M.B. y D. Menella, 2004. Requerimientos industriales de trigo en Argentina. IDIA XXI. 6:16-20.

Cuniberti, M.B., 2011. Trigo: Muestreo en pre-cosecha y clasificación calidad industrial de variedades argentinas. Temas: Alimentos. (http://inta.gob.ar/ documentos/trigo-muestreo-en-pre-cosecha-yclasificacion-calidad-industrial-de-variedadesargentinas). Consultado el 05/01/2012.

Delcour, J.; S. Vanhamel and C. De Geest, 1989. Physicochemical and functional properties of rye nonstarch polysaccharides. I. Colorimetric analysis of pentosans and their relative monosaccharide composition in fractionated (milled) rye products. Cereal Chemistry 66:107-111.

Di Rienzo J.A; F. Casanoves, M.G. Balzarini, L. Gonzalez, M.Tablada y C.W. Robledo, 2008. InfoStat, versión 2008. Grupo InfoStat, FCA, Universidad Nacional de Córdoba, Argentina.

Don, C.; W.J. Lichtendonk, J.J. Plijter and R.J. Hamer, 2003. Glutenin macropolymer: A gel formed by particles. Journal of Cereal Science 37:1-7.

Don, C.; G. Mann, F. Bekes and R.J. Hamer, 2006. HMWGS affects the properties of glutenin particles in GMP thus flour quality. Journal of Cereal Science 44:127136.

Drapron, R. and B. Godon, 1987. Role of Enzimes in Baking, In: Enzyme and their role in cereal technology, St. Paul MN, USA. Kruger, Lineback and Stauffer Editors. pp 281-324.

Edwards, N.M.; J.E. Dexter, M.G. Scanlon and S. Cenkowski, 1999. Relationship Between CreepRecovery an Dynamic Oscillatory Measurements to Durum Wheat Physical Dough Properties. Cereal Chemistry 76:638-645.

Gaines, C.S., 2000. Collaborative study of methods for solvent retention capacity profiles (AACC Method 5611). Cereal Foods World 45: 303-306.

Guttieri, M.J.; D. Brown, D. Gannon, K. O'Brien and E. Souza, 2001. Solvent retention capacities of irrigated soft white spring flours. Crop Science 41:1054-1061.

Guttieri, M.J.; R. McLea, S.P. Lanning, L.E. Talbert and E.J Souza, 2002. Assessing environmental influences on solvent retention capacities of two soft white spring wheat cultivars. Cereal Chemistry 79:880-884.

Guttieri, M.J. and E. Souza, 2003. Sources of variation in the solvent retention capacity test of wheat flour. Crop Science 43:1628-1633.

Guttieri, M.J.; C. Becker and E. Suoza, 2004. Application of Wheat Meal Solvent Retention Capacity Tests Within Soft Wheat Breeding Populations. Cereal Chemistry 81:261-266. 
IRAM (Instituto Argentino de Racionalización de Materiales). Norma IRAM 15857, 1995. Trigo. Características físicas de masas. Determinación de las propiedades reológicas. Método del alveógrafo. Buenos Aires, Argentina. (http://www.iram. org.ar). Consultado el 22/03/2012.

IRAM (Instituto Argentino de Racionalización de Materiales). Norma IRAM 15858-1, 1996. Cereales. Ensayo de panificación experimental. Método para ser usado en programas de mejoramiento de trigo. Buenos Aires, Argentina. (http://www.iram.org. ar). Consultado el 22/03/2012.

IRAM (Instituto Argentino de Racionalización de Materiales). Norma IRAM 15855, 2000. Harina de trigo. Características físicas de las masas. Determinación de la absorción de agua y de las propiedades reológicas, utilizando un farinógrafo. Buenos Aires, Argentina. (http://www.iram.org.ar). Consultado el 22/03/2012.

IRAM (Instituto Argentino de Racionalización de Materiales). Norma IRAM 15862, 2003. Cereales. Determinación de la actividad de la alfa-amilasa (número de caída), según Hagberg-Perten. Buenos Aires, Argentina. (http://www.iram.org.ar). Consultado el 22/03/2012.

IRAM (Instituto Argentino de Racionalización de Materiales). Norma IRAM 15864, 2007. Trigo y harina de trigo. Determinación de gluten húmedo, de gluten seco y de índice de gluten. Método de lavado automático. Buenos Aires, Argentina. (http://www. iram.org.ar). Consultado el 22/03/2012.

IRAM (Instituto Argentino de Racionalización de Materiales). Norma IRAM 15854, 2010. Molienda experimental para trigo pan. Parte I: Preparación y acondicionamiento de la muestra. Parte II: Método Bühler. Buenos Aires, Argentina. (http://www.iram.org. ar). Consultado el 22/03/2012.

IRAM (Instituto Argentino de Racionalización de Materiales). Norma IRAM 15853, 2012. Cereales. Método de determinación de la masa de 1000 granos. Buenos Aires, Argentina. (http://www.iram. org.ar). Consultado el 22/03/2012.

ISO (International Organization for Standardization). Norma 7971-2, 1995. Método de determinación del peso específico. Buenos Aires, Argentina.

Kulkarni, R.G; J.G. Ponte, J.R. and K. Kulp, 1987. Significance of gluten content as an Index of Flour
Quality. Cereal Chemistry 64:1-3.

Lezcano, E., 2007. Análisis de cadenas alimentarias: Informe sobre trigo y sus derivados. Secretaría de Agricultura, Ganadería, Pesca y Alimentos, Argentina.

Lezcano, E., 2010. Informe sectorial n5: Farináceos. Dirección Nacional de Transformación y Comercialización de Productos Agrícolas y Forestales; Secretaría de Agricultura, Ganadería y Pesca, Argentina.

Moiraghi, M.; L. Vanzetti, C. Bainotti, M. Helguera, A. León y G. Pérez, 2011. Relationship Between Soft Wheat Flour Physicochemical Composition and CookieMaking Performance. Cereal Chemistry 88:130-136.

Otamendi, M. A., 2004. Demandas de calidad de trigo a nivel mundial. IDIA XXI. 6:11-15.

Quaglia, G., 1991. La harina de trigo. En: Ciencia y tecnología de la panificación, España. Editorial Acribia. pp. 31-36.

Roccia, P.; M. Moiraghi, P.D. Ribotta, G.T. Perez, A. Rubiolo y A.E. León, 2006. Use of solvent retention capacity profile to predict the quality of triticale flours. Cereal Chemistry 83:243-249.

Salomón N. y R. Miranda, 2003. Índice de calidad industrial: una herramienta para determinar la aptitud de los materiales genéticos. En: Estrategias y metodologías utilizadas en el mejoramiento de trigo. Seminario Internacional de CIMMYT - INIA, Uruguay. Kohli, Ackermann y Castro Editores. pp. 163-173.

Steffolani, M.E., G.T. Pérez, P.D. Ribotta y A.E. León, 2007. Relationship between variety clasification and breadmaking quality in argentine wheats. International Journal of Agricultural Research 2: 33-42.

Weegels, P.L.; A.M. Van de Pijpekamp, A. Graveland, R.J. Hamer and J.D. Schofield, 1996. Depolymerisation and re-polymerisation of wheat glutenin during dough processing. Relationships between glutenin macropolymer content and quality parameters. Journal of Cereal Chemistry 23:103-111.

Weegels, P.L.; R.J.Hamer and J.D. Schofield, 1997. Functional properties of low Mr wheat proteins. III. Effects on composition of the glutenin macropolymer during dough mixing and resting. Journal Cereal Science 25:165-173. 